\section{Martine Raphaël Virginie Eclache Antoine Martin Jean Feuillard}

\title{
Les lymphomes du SIDA
}

Les lymphomes non hodgkiniens sont fréquents chez les malades atteints du SIDA, constituant des tumeurs de grade élevé où les cellules prolifèrent rapidement. On en distingue trois types majeurs: le lymphome de Burkitt à petites cellules non clivées, le lymphome à grandes cellules ou centroblastique et le lymphome immunoblastique. Plus rares sont les lymphoproliférations polymorphes et les lymphomes anaplasiques à grandes cellules exprimant CD30. Plus de $90 \%$ de ces lymphomes sont de type B. Comme dans les proliférations lymphocytaires des déficits immunitaires, le virus d'Epstein-Barr (EBV) joue un rôle important dans la pathogénie de ces lymphomes. L'activation et la prolifération des cellules du système immunitaire favorisent aussi la survenue de remaniements génétiques dont les plus fréquents intéressent les oncogènes $c-M Y C, B C L-6$, la famille $R A S$ et le gène suppresseur de tumeur $p 53$. Enfin, le rôle de la stimulation antigénique par le VIH dans la prolifération lymphomateuse est aujourd'hui bien documenté.

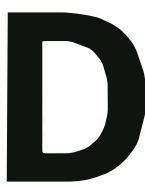
ès les premières descriptions du syndrome de déficit immunitaire acquis (SIDA), des cas de lymphomes non hodgkiniens ont été rapportés cheı des sujets homosexuels [1]. Rapidement 90 cas ont été rassemblés qui montraient un lien entre la maladie lymphomateuse ct le SII)A [1], conduisant à inclure les lymphomes B de haut grade de malignité dans la définition du SIDA. I.es études épidémiologiques ont montré que le risque de développer un lymphome non hodgkinien lors de l'infection par le virus de l'immunodéficience humaine (VIH) est de 60 à 100 fois plus élevé que dans la population générale [2]. Les lymphomes non hodgkiniens sont observés dans $5 \%$ à $10 \%$ des cas de SIDA ct représentent la première manifestation dans $3 \%$ à $4 \%$ des cas. De plus, le risque d'apparition d'un lym- phome augmente avec la prolongation de la survie [3]. Tous les groupes de sujets à risque pour l'infection à VIH peuvent développer un lymphome non hodgkinien, mais avec une fréquence variable selon les facteurs de risques: homosexuels $(3,4 \%)$, hémophiles $(5,2 \%)$, après transfusion $(3,5 \%)$, toxicomanes par voie intraveineuse $(1,6 \%)$, enfants après contamination périnatale $(1,3 \%)$, hétérosexuels, Afrique ou Caraibes ( 1 $\%$ ) [4]. Ces lymphomes non hodgkiniens sont caractérisés par leurs localisations extraganglionnaires, la fréquence des lymphomes cérébraux primitifs, le haut grade de malignité des types histologiques et la nature $\mathrm{B}$ de la prolifération tumorale. Leur pronostic est extrêmement péjoratif en raison de l'agressivité de la prolifération tumorale elle-même et du déficit immunitaire sous-jacent qui rend la conduite thérapeutique diffi- 


\section{RÉFÉRENCES}

1. Ziegler JL, Beckstead JA, Volderbin PA, Abrams DI, Levine AM, Lukes RJ, Gill PS, Burkes RL, Meyer PR, Metroka CE, et al. Non Hodgkin's lymphoma in 90 homosexual men. Relation to generalized lymphadenopathy and the acquired immunodeficiency syndrome. $N$ Engl J Med 1984; $311: 565-70$

2. Beral V, Peterman T, Berkelman R, Jaffe H. AIDS-associated non Hodgkin's lymphoma. Lancet 1991 ; 337: 805-9.

3. Gail MH, Pluda JM, Rabkin CS, Biggar RJ, Goedert JJ, Horm JW, Sondik EJ, Yarchoan $R$, Broder S. Projections of the incidence of non Hodgkin's lymphoma related to acquired immunodeficiency syndrome. $J$ Natl Cancer Inst 1991; 83: 695-701.

4. Obrams GI, Grufferman S. Epidemiology of HIV associated nonHodgkin's lymphoma. Cancer Surv 1991 ; 10: 916-22.

5. Gisselbrecht C, Oksenhendler E, Tirelli U, Lepage E, Gabarre J, Farcet JP, Gastaldi R, Coiffier B, Thyss A, Raphaël M, et al. Human immunodeficiency virus-related lymphoma treatment with intensive combination chemotherapy. AmJ Med 1993; 95 : 188-96.

6. Raphaël M, Gentilhomme O, Tulliez M and the French study group for HIV associated tumors. Histopathologic features of high grade non Hodgkin's lymphomas in acquired immunodeficiency syndrome. Arch Pathol Lab Med 1991 ; 115 : 15-20.

7. Kalter SP, Riggs SA, Cabanillas F, Butler II. Hagemester FB, Mansell PW, Newell GR, Velasquez WS, Salvador P, Barlojie B, et al. Aggressive non Hodgkin's lymphomas in immunocompromised homosexual males. Blood 1985; 66: 655-9.

8. Hessol NA, Katz MH, Liu L, Buchbinder SP, Rubino CJ, Holmberg SD. Increased incidence of Hodgkin's disease in homosexual men with HIV infection. Ann Interm Med 1992; 117: 309-11.

9. Herndier BG, Sanchez HC, Chang KL, Chen YY, Weiss LM. High prevalence of Epstein-Barr virus in the Reed-Sternberg cells of HIV-associated Hodgkin's disease. Am J Pathol 1993; 142: 1073-9.

10. Ioachim HL, Dorsett B, Cronin W, Maya M, Wahl S. Acquired immunodeficiency syndrome-associated lymphomas: clinical, pathologic, immunologic and viral characteristics of 111 cases. Hum Pathol 1991 $22: 659-73$

11. Raphael M, Audouin J, Tulliez M, et al. Anatomic and histologic distribution of 448 cases of AIDS-related non Hodgkin's lymphomas. Blood 1993; 82 : 386a. cile [5]. Selon les classifications histologiques utilisées, trois types sont décrits: à grandes cellules ou centroblastiques, immunoblastiques et de type Burkitt [6]. Ce dernier aspect est exceptionnel dans les autres types de déficits immunitaires, iatrogènes ou congénitaux. Souvent précédé d'un syndrome de polyadénopathies, le lymphome de Burkitt est plus fréquemment observé lorsque le déficit immunitaire est peu sévère [7]. Des types histologiques différents selon le degré d'immunodéficience témoignent de mécanismes physiopathogéniques multiples. Ces mécanismes font intervenir, non seulement l'activation et la dérégulation du système immunitaire liées à l'infection par le VIH, mais aussi les multiples étapes de la lymphomagenèse incluant les modifications oncogéniques et le rôle de certains virus comme le virus d'Epstein-Barr (EBV).

La maladie de Hodgkin ne sera que mentionnée, elle occupe une place particulière ; moins fréquente que les lymphomes non hodgkiniens [8], elle est caractérisée par son évolution clinique défavorable, et des localisations viscérales fréquentes. Les aspects histologiques les plus représentés sont le type scléro-nodulaire ou à cellularité mixte. C'est l'association quasiment constante avec l'EBV présent dans les cellules de Sternberg et n'exprimant que la protéine de latence LMP (latent membrane protein), comme dans le carcinome du nasopharynx, qui doit être soulignée dans cette affection [9].

Après la description des aspects anatomo-cliniques et pathologiques, nous analyserons les différents mécanismes physiopathogéniques aboutissant à la prolifération lymphomateuse.

\section{Aspects anatomo-cliniques et pathologiques des Iymphomes non hodgkiniens}

Les lymphomes non hodgkiniens du SIDA sont disséminés dans $75 \%$ des cas, intéressant préférentiellement un site extraganglionnaire. Les localisations les plus fréquentes sont digestives, cérébrales, surtout primitives et médullaires. Des localisations rarement observées dans la population générale peuvent être la manifestation initiale du lymphome non hodgkinien, comme les localisations buccales et musculaires; toutes les localisations viscérales ont pratiquement été décrites $[6,10]$. L'analyse de la série de 448 cas du Groupe français des tumeurs associées au VIH a permis d'identifier 5 groupes morphologiques [8]. Trois aspects ont été reconnus dès les premières descriptions: lymphome à petites cellules non clivées ou lymphome de Burkitt $(38 \%)$ (figures $1 A$ et $1 B)$, lymphome à grandes cellules ou lymphome centroblastique $(27 \%)$ (figure 2), lymphome immunoblastique avec ou sans différenciation plasmocytaire $(23 \%)$ (figures $3 A$ et $3 B$ ). A ces trois catégories, deux aspects doivent être ajoutés: les lymphoproliférations polymorphes, proches des aspects observés après transplantation d'organe $(6 \%)$, et les lymphomes à grandes cellules anaplasiques (4\%). Ce dernier type morphologique, aussi décrit dans la population générale, est constitué d'une prolifération de grandes cellules lymphoïdes activées. Les corrélations avec les localisations anatomiques déjà établies dans une série précédente [6] ont été confirmées. En effet, la majorité des lymphomes non hodgkiniens cérébraux primitifs sont de type immunoblastique avec différenciation plasmocytaire. Les localisations médullaires et ganglionnaires sont essentiellement représentées par le lymphome de Burkitt $(\mathrm{m} / \mathrm{s}$ $n^{\circ} 2$, vol. 3, p. 112, $n^{\circ} 4$, vol. 7, p. 390) $[6,10,11]$.

La nature B de ces lymphomes non hodgkiniens est reconnue dans plus de $90 \%$ des cas. Les études immunophénotypiques incluant les antigènes de différenciation de la lignée $\mathrm{B}$ et d'activation (HLA-DR, CD10, CD19, CD20, CD21, CD22, CD23, CD38, CD30), l'étude de l'expression des immunoglobulines de surface et intracytoplasmiques ainsi que l'analyse du réarrangement des gènes codant pour les chaînes des immunoglobulines $(\mathrm{RJH})$, ont permis d'identifier un profil immunophénotypique et moléculaire de ces lymphomes non hodgkiniens [12] résumé dans le Tableau I. Les lymphomes de Burkitt sont monoclonaux, expriment des immunoglobulines de surface, surtout $\operatorname{IgMK}$, et les antigènes de différenciation CD10, CD19, CD20, CD22 

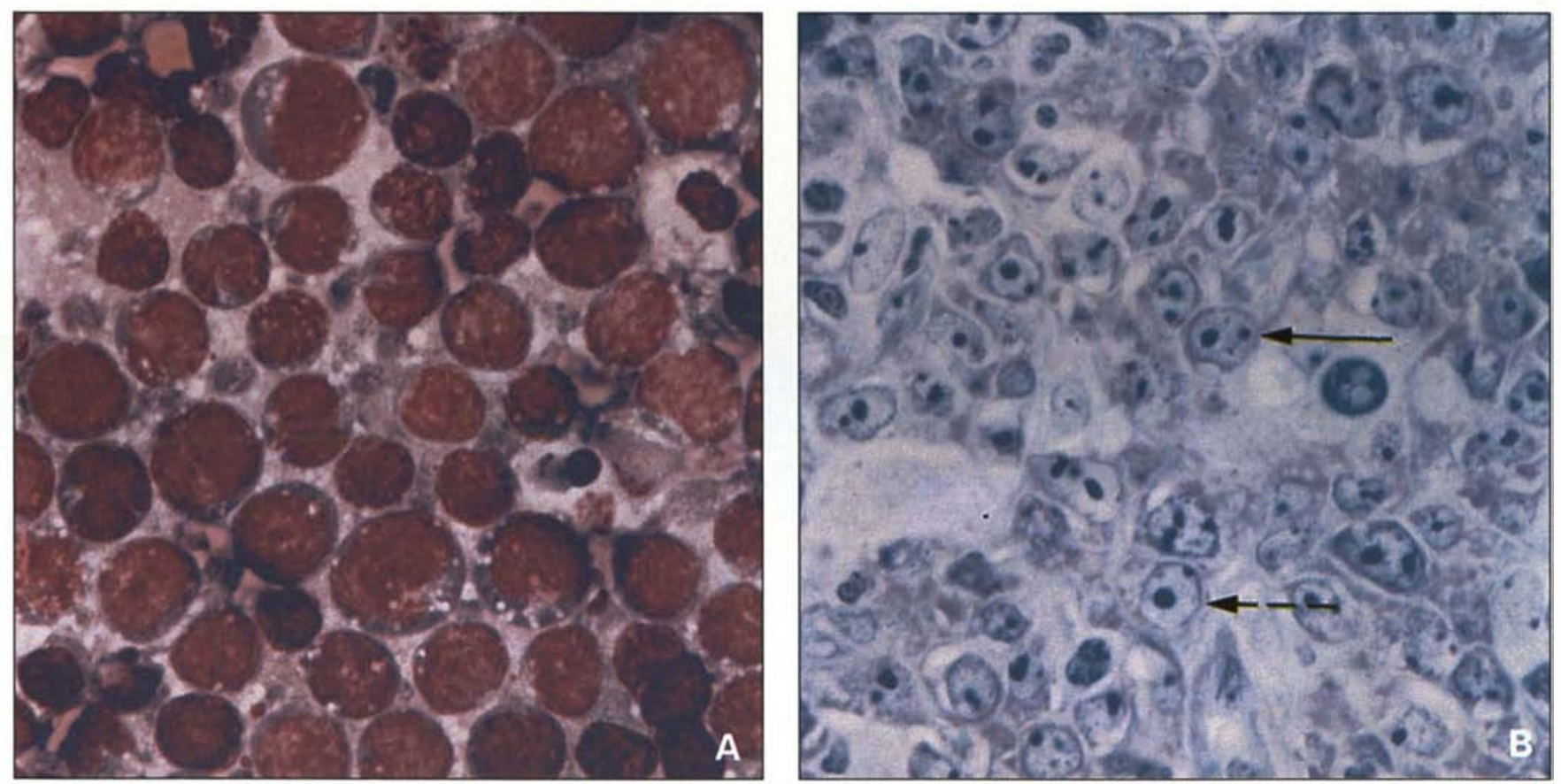

Figure 1. Lymphome de Burkitt, aspect atypique avec différenciation immunoblastique et plasmocytaire. Présence de cellules de type Burkitt: cellules de taille movenne avec plusieurs petits nucléoles $(\rightarrow)$, association avec des éléments cellulaires ayant des caractéristiques morphologiques de différenciation immunoblastique et plasmocytaire (--). A: Aspect cytologique, May Grunwald Giemsa x 1125. B: Aspect histologique, Giemsa x 500 .

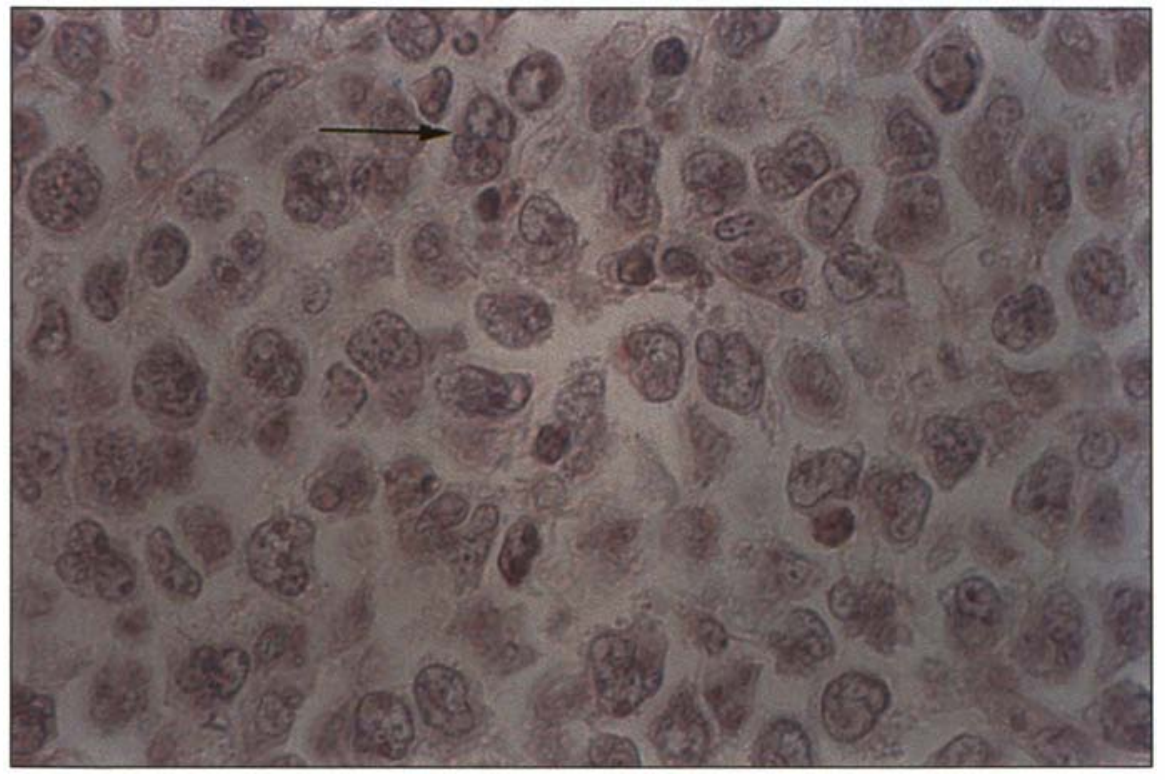

Figure 2. Lymphome à grandes cellules ou centroblastique. Présence de quelques grandes cellules à noyaux très contournés correspondant à des centroblastes multilobés $(\rightarrow$ ). Hématéine-éosine $\times 500$.

$m / s n^{\circ} 5$, vol. 11, mai 95 et CD38. Ce profil immunophénotypique est proche de celui des grandes cellules ou centroblastes d'origine centrofolliculaire. Les lymphomes non hodgkiniens immunoblastiques sont monoclonaux dans $80 \%$ des cas, expriment les marqueurs CD10, CD20 et CD38, des immunoglobulines intracytoplasmiques dans moins de $50 \%$ des cas et l'expression des immunoglobulines de surface persiste dans $90 \%$ des cas. Des cas de lymphomes immunoblastiques et de lymphoproliférations polymorphes sont oligoclonaux ou polyclonaux comme cela a été décrit après transplantation d'organe. Les lymphomes non hodgkiniens anaplasiques à grandes cellules expriment l'antigène CD30 [12]. La répartition de l'expression des molécules d'activation et d'adhérence varie selon le type histologique. L'expression de CD30, CD39, CDW70 observée sur les lymphomes à grandes cellules et immunoblastiques 


\section{RÉFÉRENCES}

12. Raphael M, Audouin J, Lamine $M$, et al. Immunophenotypic and genotypic analysis of AIDS-related non Hodgkin's lymphomas. Correlation with histologic features in 36 cases. A $m$ J Clin Pathol 1994 ; 101 : 773-82.

13. Hamilton-Dutoit SJ, Rea D, Raphaēl $M$ Sandvej K, Delecluse HJ, Gisselbrecht C, Marelle L, van Krieken HJ, Pallesen G. Epstein-Barr virus latent gene expression and tumor cell phenotype in acquired immunodeficiency syndrome-related non Hodgkin's lymphoma: correlation of lymphoma with three distinct patterns of viral latency. Am J Pathol 1993; 143: 1072-85.

14. Thomas JA, Cotter F, Hanby AM, Long LG, Morgan PR, Bramble B, Bailey BMW. Epstein-Barr virus-related oral T-cell lymphoma associated with human immunodeficiency virus immunosuppression. Blood $1993 ; 81: 3350-6$.

15. Hendier BG, Shiramizu BT, Jewett NE, Aldape KD, Reyes GR, McGrath MS. Acquired immunodeficiency syndrome-associated T-cell lymphoma: evidence for human immunodeficiency virus type 1-associated Tcell transformation. Blood 1992; 79: 1768-74.

16. Marelle L, Rea D, Raphaël M. Le virus d'Epstein-Barr et les proliférations lymphoides. médecine/sciences 1993; 9: 693-700.

17. Shibata D, Weiss LM, Nathwani BN, Brynes RK, Levine A. Epstein-Barr virus in lymph node biopsies from individuals infected with the human immunodeficiency virus is associated with concurrent or subsequent development of non Hodgkin's lymphoma. Blood 1991; 77 : 1527-33.

18. Joab I, Triki H, de Saint Martin J Perricaudet M, Nicolas JC. Detection of Epstein-Barr virus transactivator (ZEBRA) antibodies in sera from patients with human immunodeficiency virus. I Infect Dis 1991 ; $163: 53-6$.

19. Hamilton-Dutoit SJ, Raphaël $M$, Audouin J, Diebold J, Lisse I, Pedersen C, Oksenhendler F, Marelle L, Pallesen G. In situ demonstration of Epstein-Barr virus small RNAs (EBER-1) in acquired immunodeficiency syndrome-related lymphomas. Correlation with morphology and primary site. Blood 1993;82:619-24.

20. Shibata D, Weiss LM, Hernandez AM, Nathwani BN, Bernstein L, Levine A. Epstein-Barr virus-associated non Hodgkin's lymphoma in patients infected with the human immunodeficiency virus. Blood $1993 ; 81: 2102-9$.

21. Mac Mahon EE, Glass J, Hayward SD, Mann RB, Becker PS, Charache P, McArthur JC, Ambinder RP. Epstein-Barr virus in AIDS-related primary central nervous system lymphoma. Lancet 1991 ; 338 : 969-73.

22. Boyle MJ, Sewell WA, Sculley TB, Appolloni A, Turner JJ, Swanson CE, Penny $R$, Cooper DA. Subtype of Epstein-Barr virus in human immunodeficiency virus-associated non Hodgkin's lymphoma. Blood 1991; est absente dans les lymphomes de Burkitt. L'expression des molécules d'adhérence LFAl (CD1la, CD18), ICAM-1 (CD54), LFA3 (CD58) est diminuée, voire absente, dans les lymphomes de Burkitt, comme cela avait été rapporté dans les cas observés chez les sujets immunocompétents. En revanche, l'expression est variable dans les lymphomes à grandes cellules et immunoblastiques où ces molécules sont souvent exprimées, mais leur expression peut être dissociée [13].

Les lymphomes cérébraux primitifs occupent une place particulière, par le caractère relativement homogène de leur aspect histologique. En effet tous les cas de notre série sont caractérisés par l'importance du contingent immunoblastique avec différenciation plasmocytaire. L'expression des antigènes de différenciation de la lignée B est dissociée; en revanche, les molécules d'activation et d'adhérence sont présentes [13].

De rares cas de lymphomes $\mathrm{T}$ ont été observés au cours de l'infection par le $\mathrm{VIH}$, incluant différentes catégories de proliférations lymphomateuses de type $\mathrm{T}$ périphérique comme des proliférations angiocentriques et des lymphomes à grandes cellules anaplasiques. Les lymphomes $T$ peuvent être associés à l'EBV [14] et l'intégration du VIH a été démon trée dans un cas [15].

\section{Les mécanismes physiopathogéniques}

Plusieurs mécanismes physiopathogéniques interviennent dans les lymphomes non hodgkiniens B du SIDA. Les mécanismes principaux impliquent le rôle de l'EBV et les anomalies moléculaires. En outre, le dysfonctionnement immunitaire par la diminution de l'immunosurveillance des cellules infectées par l'EBV et par la stimulation chronique des lymphocytes B favorise l'apparition de la prolifération lymphomateuse. L'incidence de ces mécanismes varie selon le degré du déficit immunitaire, la localisation et le type histologique du lymphome non hodgkinien.

\section{L'EBV et les lymphomes non}

hodgkiniens du SIDA

L'EBV, virus ubiquitaire de la famille des Herpes viridae capable d'infecter des cellules épithéliales de l'oropha- rynx et les lymphocytes, est l'agent responsable de la mononucléose inf ectieuse lors de la primo-inf ection chez l'homme (voir [16] pour revue générale). Le rôle prépondérant de l'EBV dans la pathogénie des lymphomes non hodgkiniens du SIDA a été suggéré en raison du lien connu entre l'EBV et les proliférations lymphoïdes des déficits immunitaires [16], de la mise en évidence de l'EBV dans les ganglions des hyperplasies folliculaires du syndrome de polyadénopathies pouvant précéder le lymphome [17], et par la présence de signes de réactivation dans le sérum avec la détection d'anticorps dirigés contre les protéines de réplication EA (early antigen), VCA (viral capsid antigen) et ZEBRA (Z Epstein-Barr replication activator) [18].

Les analyses comparatives entre les aspects histologiques, les localisations et la détection de l'EBV par les techniques d'amplification génique (PCR) et d'hybridation in situ utilisant la sonde EBER1 (EBV encoded small $R N A s)$ ont démontré la présence de l'EBV dans $66 \%$ des cas de lymphome non hodgkinien du SIDA avec une répartition différente selon le type histologique et la localisation $[19,20]$. L'EBV est présent dans $90 \%$ à $100 \%$ des cas de lymphomes cérébraux primitifs [21], il est observé dans $17 \%$ des lymphomes non hodgkiniens à grandes cellules, $66 \%$ des lymphoprolif érations polymorphes, $70 \%$ à $80 \%$ des lymphomes immunoblastiques et $34 \%$ des cas de lymphome de Burkitt. La présence de l'EBV dans $34 \%$ des cas de lymphome de Burkitt est une situation particulière aux lymphomes du SIDA. En effet, dans les lymphomes de Burkitt endémiques ou africains, l'EBV est associé dans $95 \%$ des cas; en revanche, dans les lymphomes de Burkitt non endémiques ou sporadiques, l'EBV n'est présent que dans $10 \%$ à $15 \%$ des cas. Les deux types $A$ et $B$ de l'EBV, différenciés par l'antigène EBNA-2 (Epstein Barr nuclear antigen), sont observés, mais la prévalence du type $B$ est élevée comme dans le lymphome de Burkitt endémique [22]. L'analyse des séquences répétées terminales en utilisant la sonde Xhol a montré le caractère homogène, dit "clonal ", des séquences répétées terminales, suggérant que l'EBV a précédé la prolifération 


\begin{tabular}{|c|c|c|c|c|c|c|c|c|c|}
\hline \multicolumn{10}{|c|}{$\begin{array}{l}\text { ASPECTS MORPHOLOGIQUES, IMMUNOPHÉNOTYPIQUES } \\
\text { ET GÉNOTYPIQUES DES TROIS PRINCIPAUX TYPES HISTOLOGIQUES DES LYMPHOMES DU SIDA }\end{array}$} \\
\hline $\begin{array}{l}\text { Morphologie } \\
\text { Type histologique }\end{array}$ & (11) Fréquence* & CD10* & $\underset{\text { CD19* }}{\operatorname{Immu}}$ & CDhénot & CD22* & CD38* & $\underset{\text { clg* }^{*}}{\operatorname{Immu}}$ & $\begin{array}{l}\text { globuli } \\
\text { slg* }\end{array}$ & $\begin{array}{l}\text { 1es } \\
\text { R(JH)* }\end{array}$ \\
\hline Lymphome de Burkitt & 38 & 75 & 60 & 65 & 75 & 75 & 20 & 100 & 100 \\
\hline $\begin{array}{l}\text { Grandes cellules ou } \\
\text { centroblastique }\end{array}$ & 27 & 100 & 100 & 100 & 100 & 0 & 66 & 100 & 100 \\
\hline Immunoblastique & 23 & 60 & 10 & 50 & 10 & 70 & 70 & 80 & 60 \\
\hline
\end{tabular}

(1) d'après [8].

(2) d'après [12].

"pourcentage de cas positifs.

clg: immunoglobuline cytosolique; slg: immunoglobuline de surface.

$R(\mathrm{JH})$ : réarrangement de la région de jonction $(\mathrm{JH})$ du gène des chaînes lourdes des immunoglobulines.
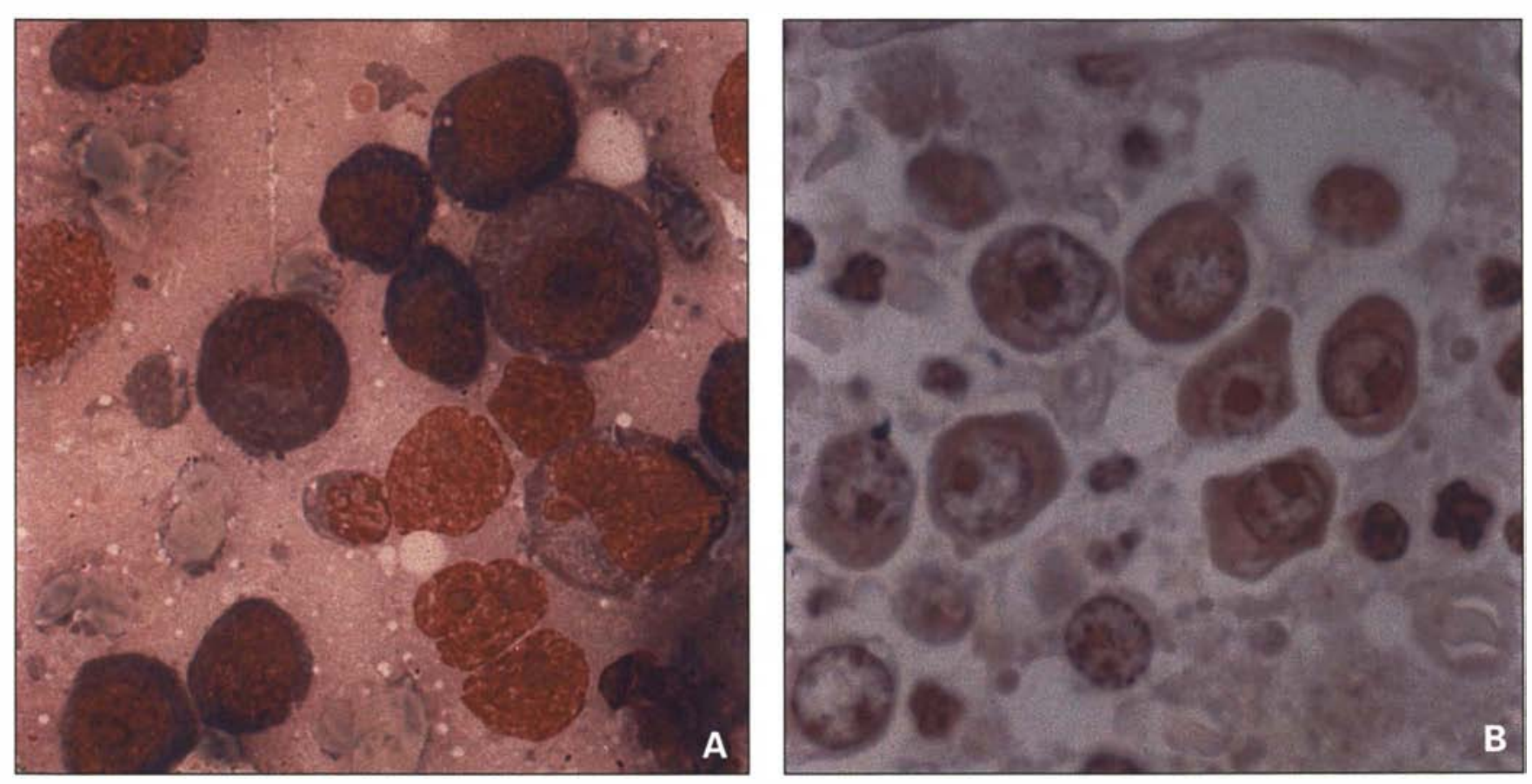

Figure 3. Lymphome immunoblastique avec différenciation plasmocytaire. A: aspect cytologique, May Grunwald Giemsa x 1125. B: aspect histologique, hématéine-éosine $\times 1125$.

lymphomateuse, ce qui renforce l'hypothèse du rôle pathogénique de ce virus [23].

L'analyse de l'expression des protéines de latence, dont LMP (latent membrane protein) et EBNA-2 connues pour leur activité transformante et proliférative au sein des cellules infectées et celle des modifications immunophénotypiques cellulaires liées à la présence du virus, est une des étapes de la compréhension du rôle de $m / s n^{\circ} 5$, vol. 11, mai 95
l'EBV dans la lymphomagenèse. Les trois types de latence de l'EBV sont décrits: latence I où seule la protéine EBNA-1 est présente, latence II où les protéines EBNA-1 et LMP sont exprimées et latence III où les 9 protéines EBNA: 1, 2, 3A, 3B, 3C, LP et les LMP : 1, 2A/2B seraient exprimées comme dans certaines lignées lymphoblastoïdes [24]. Des corrélations ont pu être établies entre les types de latence et les localisations. Le type I de latence est observé dans le groupe des lymphomes de Burkitt comme cela est classiquement décrit. Toutefois, dans notre série, trois cas expriment LMP; cette expression inhabituelle de LMP dans ce type de lymphome est probablement liée au déficit immunitaire. Les types I, II et III sont présents dans les lymphomes à grandes cellules et immunoblastiques. La comparaison avec les localisations a montré une plus grande 


\section{RÉFÉRENCES}

23. Gulley ML, Raphaël M, Lutz CT, Ross DW, Raab-Traub N. Epstein-Barr virus integration in human lymphomas and lymphoid cell lines. Cancer 1992; 70:185-91.

24. Kieff E, Liebowitz D. Epstein-Barr virus and its replication. In: Fields BN, Knipe DM, et al. eds. Virology, 2nd ed. New York: Raven Press, 1990: 1889-920.

25. Rea D, Delecluse HJ, Hamilton-Dutoit SJ, et al. EBV latent and replicative gene expression in post-transplant lymphoproliferative disorders and AIDS-related non Hodgkin's lymphomas. Ann Oncol 1994; 5 (suppl 1) : S113-6.

26. Birx DL, Redfield RR, Tosato G. Defective regulation of Epstein-Barr virus infection in patients with acquired immunodeficiency syndrome (AIDS) or AIDS- related disorders. N Engl J Med 1986; 314: 874-9.

27. Brousset P, Drouet E, Schlaifer D, et al. Epstein-Barr virus replicative gene expression in tumour cells of AIDS-related non sion in tumour cells of AIDS-related non number and antibody titres to EBV. AIDS $1994 ; 8: 583-90$.

28. Ballerini P, Gaidano G, Gong JZ, Tassi V, Saglio G, Knowles DM, Dalla-Favera R. Multiple genetic lesions in acquired immunodeficiency syndrome-related non Hodgkin's lymphoma. Blood 1993; 81 : 166-76.

29. Magrath I, Jain V, Bhatia K. Molecular epidemiology of Burkitt's lymphoma. In: Tursz T, Pagano JS, Ablashi DV, De Thé G, Lenoir $\mathrm{G}$, Pearson GR, eds. The Epstein-Barr virus and associated diseases. Paris: Inserm/ John Libbey Eurotext, 1993; 225 : 377-96.

30. Delecluse HJ, Raphaël M, Magaud JP, Felman P, Alsamad IA, Bornkamm GW, Lenoir GM. Variable morphology of human immunodeficiency virus-associated lymphomas with $c-M Y C$ rearrangements. Blood $1993 ; 82$ : $552-63$.

31. Lo Coco F, Ye BH, Lista F, et al. Rearrangements of the $B C L-6$ gene in diffuse large cell non Hodgkin's lymphoma. Blood $1994 ; 83$ : $1757-9$.

32. Gaidano G, Lo Coco F, Ye BH, et al. Rearrangements of the $B C L-6$ gene in acquired immunodeficiency syndrome associated non Hodgkin's lymphoma : association with diffuse large cell subtype. Blood 1994; 84: 397-401.

33. Nakamura $H$, Saïd JW, Miller CW, Koeffler HP. Mutation and protein expression of $p 53$ in acquired immunodeficiency syndrome-related lymphomas. Blood 1993; 82 : 920-6.

34. Gaidano $G$, Ballerini P, Gonj JZ, Inghirami G, Neri A, Newcomb EW, Magrath IT, Knowles DM, Dalla-Favera R. p53 mutation in human lymphoid malignancies: association with Burkitt's lymphoma and chronic lymphocytic leukemia. Proc Natl Acad Sci USA fréquence des types III dans les localisations extraganglionnaires [13]. Ces types de latence sont le reflet de l'utilisation de différents promoteurs $\mathrm{Wp} / \mathrm{Cp}$ pour la latence III comme dans les lignées lymphoblastoïdes, Fp pour les types de latence I et II comme dans le lymphome de Burkitt, le carcinome du nasopharynx et la maladie de Hodgkin. La rupture de la latence et l'entrée dans le cycle réplicatif ont été mises en évidence dans $36 \%$ des cas de lymphomes non hodgkiniens à grandes cellules et/ou immunoblastiques et dans $22 \%$ des cas de lymphome de Burkitt par l'analyse immunohistochimique de l'expression de la protéine ZEBRA [25]. Cependant, les protéines tardives du cycle lytique n'ont pas été observées, suggérant que l'entrée dans le cycle réplicatif ne conduit pas à une production abondante de virions pouvant recruter d'autres cellules lymphoïdes. Le déficit immunitaire avec la perte de l'immunosurveillance des cellules infectées par l'EBV est l'hypothèse la plus vraisemblable pour expliquer l'entrée de l'EBV dans le cycle lytique au sein de cellules lymphoïdes [26]. Toutefois, il n'a pas été démontré de relation entre le degré de déficit immunitaire, le titre des anticorps anti-EBV dans le sérum et l'expression des protéines réplicatives au sein de la tumeur [27].

\section{Les anomalies moléculaires}

Les processus moléculaires de lymphomagenèse des lymphomes non hodgkiniens du SIDA impliquent des anomalies génétiques multiples dont les plus fréquentes intéressent les oncogènes $c-M Y C, B C L-6$ et de la famille $R A S$ ainsi que le gène suppresseur de tumeur p53. L'étude des oncogènes de la famille RAS n'a porté que sur quelques cas, montrant des anomalies, phénomènes non décrits dans les processus lymphomateux de la population immunocompétente. Le réarrangement de l'oncogène $B C L-6$, localisé sur le chromosome 3 (3q27), a été montré récemment sur les lymphomes à grandes cellules et immunoblastiques.

\section{Réarrangements de l'oncogène $c$-myc}

L'expansion du compartiment des lymphocytes B et leur activation polyclonale augmente significativement la probabilité de survenue d'événements géniques comme les translocations $\mathrm{t}(8 ; 14) \quad(\mathrm{q} 24 ; \mathrm{q} 32), \mathrm{t}(8 ; 22)$ (q24;q11 ou q12), t(2;8) (p12;q24) impliquant le locus de l'oncogène $c-M Y C$ et le réarrangement préférentiel de la région de commutation isotypique dite région switch $(s \mu) \mathrm{du}$ gène des chaînes lourdes des immunoglobulines $(\mathrm{IgH})$ sur le chromosome 14 ou bien le gène codant pour la chaîne kappa sur le chromosome 2, ou le gène codant pour la chaîne lambda sur le chromosome 22. Pour les cas de lymphome de Burkitt analysés, le réarrangement de l'oncogène $c-M Y C$ a été mis en évidence [2830]. Ce réarrangement est dans la majorité des cas similaire à celui du lymphome de Burkitt non endémique intéressant sur le chromosome 8 la zone du premier intron-premier exon ou les séquences adjacentes du gène $c-M Y C$ et la région $S \mu$ sur le chromosome 14 pour le gène (IgH). Ce type de réarrangement intervient tardivement dans le développement des cellules B, c'est-à-dire lors de la commutation isotypique. Pour quelques cas, le réarrangement est identique à celui du lymphome de Burkitt endémique où le point de cassure sur le chromosome 8 est éloigné en 5' de $c-M Y C$ avec un réarrangement préférentiel dans la région de jonction $(\mathrm{JH})$ du gène $\operatorname{IgH}[28]$ (figure 4). Ce réarrangement intervient précocement dans l'ontogénie des cellules B impliquant le système des recombinases. La juxtaposition des régions enhancer du gène $\operatorname{IgH}$ est le mécanisme principal de l'activation de $c-M Y C$. Récemment, le rôle de la protéine EBNA-1 dans l'augmentation de l'activité des enhancers du gène $I g H$ a été démontré. Cette démonstration renforce l'importance du rôle de l'EBV et son intrication dans les mécanismes moléculaires de la lymphogenèse [29].

Parmi les lymphomes non hodgkiniens du SIDA ayant un profil moléculaire de lymphome de Burkitt, des aspects morphologiques de type immunoblastique avec différenciation plasmocytaire ont été identifiés, associés à la persistance de certains critères morphologiques du lymphome de Burkitt. Parmi ces critères, la cohésion des cellules et la présence d'un contingent d'au moins $10 \%$ de petites cellules non clivées de type 


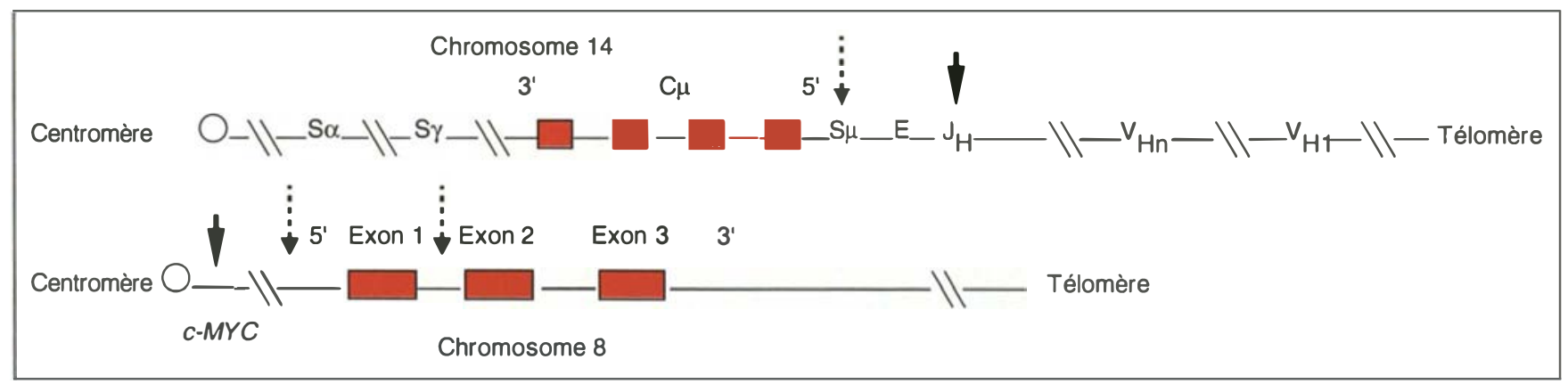

Figure 4. Réarrangement de c-MYC. Schéma représentant les différents points de cassure de c-MYC et les réarrangements préférentiels avec le gène IgH des immunoglobulines. Les flèches discontinues montrent les sites de jonction sur le chromosome 14 (gène $\mathrm{lgH}$ ) et le chromosome 8 (gène c-MYC), du réarrangement le plus fréquemment observé dans les lymphomes de Burkitt non endémiques et ceux du SIDA. Les flèches continues montrent les sites de jonction décrits dans les lymphomes de Burkitt endémiques et dans quelques cas de lymphomes associés au SIDA.

Burkitt sont reconnues. Pour ces cas, tous associés à l'EBV, la mise en évidence d'un continuum morphologique entre les petites cellules non clivées du lymphome de Burkitt et des immunoblastes avec différenciation plasmocytaire pourrait correspondre à l'un des aspects des proliférations lymphomateuses observées au cours du SIDA [30].

\section{Les mutations de RAS}

Dans les hémopathies lymphoïdes, l'activation des proto-oncogènes de la famille RAS par mutation ponctuelle au niveau des codons 12,13 et 61 n'est observée que dans deux types de maladies: la leucémie aiguē lymphoblastique et le myélome. Dans les lymphomes non hodgkiniens de la population générale, il n'a pas été détecté de mutations; en revanche, dans une étude portant sur 27 cas de lymphomes non hodgkiniens du SIDA, $15 \%$ des cas possédaient une mutation aux codons 12 ou 61 de $\mathrm{N}-R A S$ ou K-RAS. Ces anomalies correspondaient à trois cas de lymphome de Burkitt et à un cas de lymphome immunoblastique [28]. Cette analyse pourrait être étendue à de plus importantes séries et comparée aux aspects de différenciation plasmocytaire.

\section{Le réarrangement de l'oncogène $B C L 6$}

Les réarrangements de l'oncogène $B C L-6$ ont été récemment décrits dans $40 \%$ des lymphomes à grandes cellules et immunoblastiques de la population générale [31]. Dans une étude de 40 cas de lymphomes du SIDA, les réarrangements de $B C L-6$ n'ont été observés que dans $20 \%$ des cas de lymphomes à grandes cellules ou immunoblastiques. Ces réarrangements étaient isolés, sans autres anomalies génétiques associées telles que le réarrangement de $c-M Y C$, ou les mutations de la famille $R A S$ ou de p53 [32].

Inactivation du gène suppresseur de tumeur $p 53$

Le gène suppresseur de tumeur $p 53$ situé sur le chromosome 17 (17p13) code pour une phosphoprotéine nucléaire qui a la propriété d'un facteur transcriptionnel. Dans de nombreux types de cancers, la protéine peut être inactivée par perte d'hétérozygotie du locus $p 53$ et mutation sur un des domaines de la partie codante de l'allèle restant entre l'exon 5 et l'exon 9. Dans les lymphomes non hodgkiniens, $p 53$ inactivée pourrait participer à la progression tumorale comme pour d'autres tumeurs. Deux études récentes ont montré une incidence élevée de mutations de $p 53$ dans les lymphomes de Burkitt [28] et immunoblastiques [33] du SIDA. Mais sur ces petites séries, il apparaît que cette incidence n'est pas significativement différente de celle de la population immunocompétente pour les mêmes types de lymphomes non hodgkiniens
[34]. Pour l'analyse de $p 53$ dans les lymphomes non hodgkiniens, l'absence de corrélation entre une expression anormale de la protéine détectée par immunohistochimie et la présence d'une mutation doit être soulignée [33]. On peut s'interroger sur d'autres mécanismes d'inactivation de la protéine, par exemple par interaction avec une autre protéine cellulaire ou une protéine virale, et il a été montré in vitroque la protéine $\mathrm{p} 53$ peut interagir avec la protéine de latence EBNA-5 de l'EBV [35]. Dans les lymphomes non hodgkiniens du SIDA, le lien entre l'EBV et la protéine p53 reste à préciser.

\section{Le dysfonctionnement du système} immunitaire

Le déficit de l'immunité à médiation cellulaire par l'élimination des lymphocytes T CD4 diminuant les capacités d'immunosurveillance des cellules infectées par l'EBV et de défense antitumorale [26] est l'un des facteurs favorisant l'apparition des lymphomes. La recherche in situ au sein de la prolifération lymphomateuse de la présence de cellules cytotoxiques a montré qu'il n'y avait pas de déficit quantitatif significatif, suggérant ainsi l'intervention d'anomalies qualitatives [36]. L'activation polyclonale des lymphocytes $\mathrm{B}$ résultant de l'action d'agents infectieux (EBV, cytomégalovirus, action directe des déterminants antigéniques du VIH) $[26,37]$ et de la dérégulation 


\section{RÉFÉRENCES}

35. Szekely L, Selivanova (;, Magnusson K Klein G, Wiman KG. EBNA-5 an EpsteinBarr virus encoded nuclear antigen binds to the retinoblastoma and 553 proteins. Cell Biol $1993 ; 90: 5455-9$.

36. Devergne O, Raphaël M, Autran B, et al. Intratumoral activation of CD8 ${ }^{*}$ cvtotoxic lymphocyles in AIDS lymphomas. Hum Pathol 1995 (sous presse).

37. Amadori A, Zamarchi R, Veronese ML, Panozzo M, Barelli A, Borri A, Sironi M, Colotta F, Mantovani A, Chieco-Bianchi L. B-cell activation during HIV-1 infection. II Cell to cell interactions and cytokine requirement. J Immunol 1991 ; $145: 57-62$.

38. Birx DL, Redfield RR, Tencer $k$ Fowler A, Burke DS, Tosato G. Induction of interleukin-6 during human immunodeficiency virus infection. Blood $1990 ; 76: 2303$ 10.

39. Burdin N, Peronne C, Banchereau J, Rousset $F$. Epstein-Barr virus transformation induces B lymphocytes to produce human interleukin-10. I Exp Med 1993; 177: 295304.

40. Emilie D, Coumbaras I, Raphaèl M, Devergne $\mathrm{O}$, Delecluse HJ, Gisselbrecht $\mathrm{C}$ Michiels JF, van Damme J. Toga T, Kishimoto $T$, et al. Interleukin- 6 production in high grade B lymphomas: correlation with the presence of malignant immunoblasts in acquired immunodeficiency syndrome and in human immunodeficiency virus negative patients. Blood 1992; 80: 498-504.

41. Emilie D. Touitou R, Raphaël M, Peuchmaur M, Devergne $O$, Réa D, Coumbraras J, Crevon MC, Edelman L, Joab I, et al. In vivo production of interleukin-10 by malignant cells in AIDS lymphomas. Eur I Immunol 1992 ; 22 : 2937-42.

42. Laurence J, Astrin S. Human immunodeficiency virus induction of malignant transformation in human B lymphocytes. Proc Natl Acad Sci USA 1991; 88: 7635-9.

43. Gras G, Richard Y, Roques P, Olivier R, Dormont $D$. Complement and virus-specific antibody-dependent infection of normal $B$ lymphocytes by human immunodeficiency virus type 1. Blood 1993; 81: 1808-18.

44. Shirai A, Cosentino M, Leitman-Klinman SF, Klinman M. Human immunodeficiency virus infection induces both polyclonal and virus-specific B cell activation. J C.lin Invesi $1992 ; 89: 561-6$

45. Ng VL, Hurt MH, Fein CL, el al. IgMs produced by two acquired immune deficiency syndrome lymphoma cell lines: Ig binding specificity and $\mathrm{VH}$-gene putative somatic mutation analysis. Blood 1994; 83: 1067-78.

46. Riboldi P, Gaidano G, Schettino EW, al. Two acquired immunodeficiency lymphoma-associated Burkitt's lymphomas produce specific anti-i IgM cold agglutinins using somatically mutated VH $4-21$ seg-

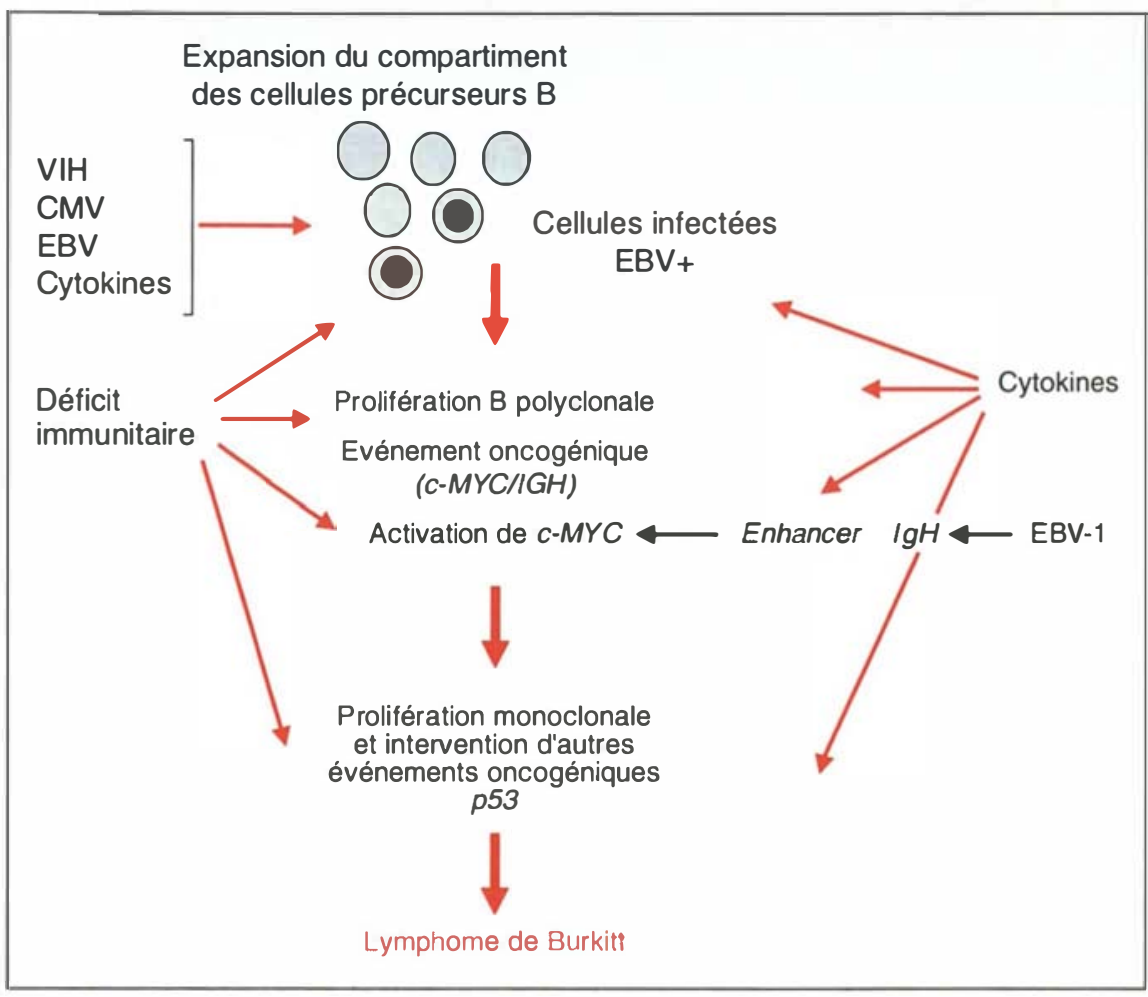

Figure 5. Physiopathologie du Iymphome de Burkitt du SIDA. L'expansion polyclonale de cellules $B$ infectées ou non par le virus EBV est liée à différents agents infectieux $(V I H$, virus de l'immunodéficience humaine, EBV, virus d'Epstein-Barr, CMV, cytomégalovirus) et à l'action de cytokines. Le déficit immunitaire permet l'accroissement de ce compartiment, augmentant la probabilité de survenue d'anomalies géniques, en particulier de la translocation t $(8 ; 14)$ impliquant c-MYC et le gène IgH des immunoglobulines. Ces anomalies géniques aboutissent à la prolifération monoclonale de cellules $B$ porteuses de la translocation $1(8 ; 14)$ caractérisant le lymphome de Burkitt. $D^{\prime}$ autres événements oncogéniques comme la mutation de p53 ou de RAS peuvent intervenir. Dans ce type de prolifération associée ou non à l'EBV, ce sont les altérations géniques qui prédominent. EBNA-1: Epstein-Barr nuclear antigen.

de la production de cytokines [38] est un autre facteur farorisant en permettant la survenue des événements oncogéniques sur une courte période de temps. Parmi les facteurs contribuant à la production de cytokines, le VIH stimule la production de TNF $\alpha$ et de l'II.6 et l'EBV celle de l'IL.6 et de l'ILl 0 [39]. Les techniques d'hybridation in situ ont permis de montrer l'accumulation de transcrits IL.6 et IL.10 dans les cellules tumorales $[40,41]$. De plus, la corrélation de la production de l'IL.6 aux aspects histopathologiques a montré l'importance de l'expression dans les lymphomes immunoblastiques avec différenciation plasmocytaire [40]. Cette corrélation à justifié la mise en route d'un protocole thérapeutique utilisant un anticorps monoclonal anti-
IL.6. Arec la même technique d'hybridation in silu, la production de l'IL.10 par les cellules tumorales lorsque l'EBV est présent a été démontrée [41]. Cette production a pour conséquence l'entretien de la prolifération lymphomateuse, liée aux propriétés de facteur de croissance pour les lymphocytes B de cette cytokine et à son action immunosuppressive, inhibant l'activation macrophagique et la production d'interféron $\gamma$.

\section{Rôle du VIH?}

Le rôle indirect du VIH par le déficit immunitaire et par l'activation polyclonale B qu'il induit est bien établi. A l'inverse, in vivio, le rôle direct du VIH dans la lymphomagenèse n'est pas démontré. In vitro, l'infection par le VIH 


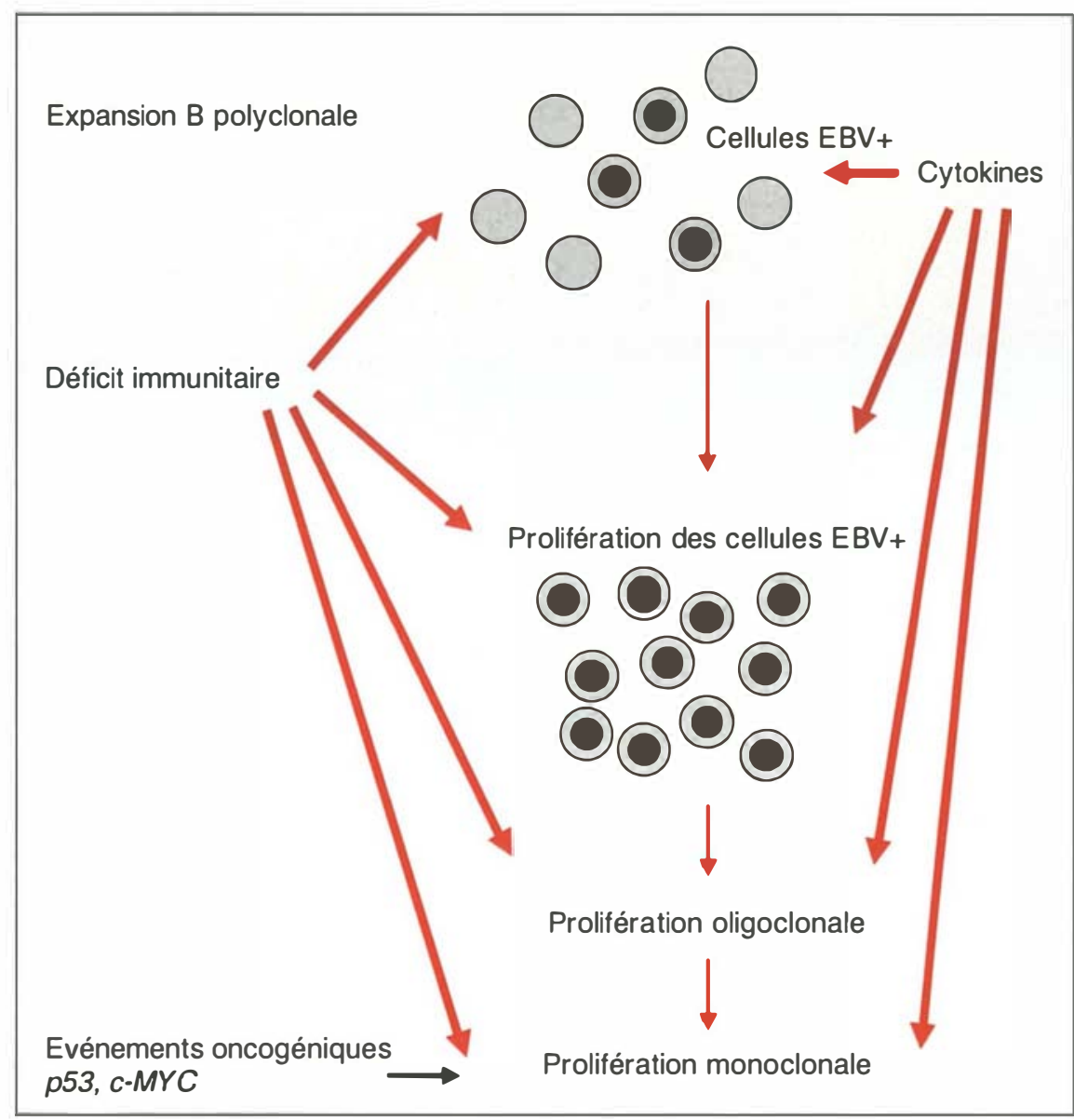

Figure 6. Physiopathologie des Iymphomes immunoblastiques du SIDA. Dans cette catégorie de lymphomes non hodgkiniens, le déficit immunitaire est majeur. La perte de l'immunosurveillance des cellules lymphocytaires $B$ infectées par l'EBV permet la prolifération polyclonale de cellules infectées par l'EBV et l'émergence progressive d'un clone malin comme cela a pu être démontré chez la souris SCID. Les événements géniques pouvant intéresser c-MYC et BCL-6 peuvent être impliqués, mais les anomalies géniques intervenant dans ce type de lymphome non hodgkinien ne sont qu'incomplètement connues. Le rôle majeur du déficit immunitaire de l'infection par l'EBV dans ces lymphomes non hodgkiniens les rapproche des lymphoproliférations et lymphomes non hodgkiniens observés lors du déficit immunitaire iatrogène après transplantation d'organe et traitement immunosuppresseur.

de lymphocvtes B infectés par l'EBV a été obtenue, aboutissant à la présence d'un contingent minoritaire de cellules devenant tumorales avec le phénotype d'un lymphome de Burkitt, exprimant fortement l'EBV et l'oncogène c-MrC: L La greffe de ce lymphome chez la souris SCID a montré une modification morphologique de type immumoblastique similaire à celle observée dans les lymphomes non hodgkiniens du SIDA [42]. Un autre modèle d'infection in vilro a permis l'infection des lymphocrtes B normaux par la mise en jeu des récepteurs du complément (.RI (C.D35) et de Burkitt de sujets infectés par le VIH a permis de montrer l'activité des immunoglobulines produites: antigpl60 pour l'une et anti-facteur rhumatoïde pour l'autre. L'utilisation préférentielle de gènes siège de mutations somatiques témoigne d'une prolifération lymphomateuse liée à une stimulation antigénique $[45,46]$.

\section{Conclusion}

Les lymphomes non hodgkiniens du SIDA sont caractérisés par leur complexité reflétant, non seulement les mécanismes intrinsèques multiples de la lymphomagenèse qui fait intervenir au niveau génomique les virus et les modifications oncogéniques, mais aussi les facteurs cellulaires. Ces facteurs internes, ou externes à la cellule comme les crtokines, interviennent par des mécanismes autocrine ou paracrine. Le contexte de dysfonctionnement immunitaire modifiant le comportement des cellules lymphoïdes non tumorales ou tumorales en réponse à l’infection par l'EBV et à la stimulation antigénique du VIH augmente le degré de complexité physiopathologique. Ainsi, deux aspects peuvent être schématiquement décrits, le premier aboutissant au lymphome de Burkitt, le second aux lymphomes à grandes cellules et immumoblastiques. Dans le premier cas, les événements oncogéniques sont prédominants, intéressant surtout les gènes $c$-MYC et p53 (jigure 5). Dans le second cas, l'importance du déficit immunitaire, l'EBV et la production de cytokines jouent un rôle prédominant (figure 6). Cette complexité et la gravité du pronostic de ces lymphomes montrent l'importance de la caractérisation morphologique, immunophénotypique, génotypique et physiopathologique très précise. Cette approche des mécanismes de la lymphomagenèse est primordiale dans cette affection en raison des difficultés majeures de traitement liées au déficit immunitaire imposant une stratégie thérapeutique de plus en plus spécifique

\section{TIRÉS À PART}

M. Raphaēl. 


\section{Summary}

AIDS-associated lymphoma

The overall risk of non Hodgkin's lymphoma (NHL) with AIDS is about 60 to 100 times more common than in the general population. Five to $10 \%$ of patients with HIV infection develop a lymphoma, in about $4 \%$ as primary AIDS manifestation. Gastrointestinal tract (GI-tract), central nervous system, bone marrow and oral cavity are the most frequent extra-nodal sites. Three main histologic categories are identified: small noncleaved cell lymphoma or Burkitt's lymphoma, large noncleaved cell or centroblastic lymphoma and immunoblastic lymphoma with plasmocytic features. In addition, two other aspects are described: polymorphic lymphoproliferation close to post transplant lymphoproliferative disorders and anaplastic large cell lymphoma expressing CD30 antigen. Regarding immunophenotypic and genotypic aspects, more than $90 \%$ of cases are B-cell type. Burkitt's lympho- mas are monoclonal, associated with EBV in $30 \%$ to $40 \%$ of cases. Most immunoblastic cases are monoclonal, but some cases are polyclonal. Systemic immunoblastic NHL cases are associated with EBV in $70 \%$ to $80 \%$ of cases, while in primary central nervous system NHL, EBV is nearly always present. Activation and dysregulation of the immune system with cytokines production leads to the expansion and the activation of B cells, increasing the probability of occurrence of genetic lesions in a relative short period of time. $c-M Y C, B C L-6$ and $R A S$ family oncogenes and $p 53$ tumor suppressor gene modifications occur. The frequent translocation $\mathrm{t}(8 ; 14)$ involving $c-M Y C$ is mostly similar to those observed in non endemic Burkitt's lymphoma. At the molecular level, Burkitt's lymphomas are characterized by $c-M Y C$ rearrangements associated with mutations of RAS family oncogenes and p53 in some cases. Large cells and immunoblastic lymphomas are associated with $B C L-6$ rearrangements in $20 \%$ of cases. The exact role of HIV in AIDS-related NHL remains unclear. In vitro, EBV inf ected or normal B-cells can be infected by HIV but this was not clearly demonstrated in vivo. However, direct B-cell stimulation by antigen was observed in several AIDS-related NHL cell lines. AIDS-related NHL of B-cell type are one of the most complex lymphomatous proliferations where several pathogenic mechanisms are intricated such as immune dysregulation, cytokines production (mainly IL6 and IL10), genetic lesions of lymphomagenesis and EBV. Two main categories are distinguished: Burkitt's lymphomas where genetic lesions are predominant and the category of large cell/ immunoblastic lymphoma where immunosuppression, EBV and probably $B C L-6$ rearrangements are playing a role. 